\title{
ANÁLISE DA VIABILIDADE ECONÔMICO-FINANCEIRA DA ENERGIA SOLAR FOTOVOLTAICA EM UMA INSTITUIÇÃO DE ENSINO SUPERIOR NO ESTADO DO CEARÁ
}

\author{
Cícero Allan Barbosa Soares ${ }^{1}$ \\ Jeniffer de $\mathrm{Nadae}^{2}$ \\ Diego Coelho do Nascimento ${ }^{3}$
}

\begin{abstract}
RESUMO
O presente estudo teve como objetivo a análise da viabilidade econômico-financeira da instalação de um sistema gerador de energia solar fotovoltaica em uma Instituição de Ensino Superior (IES) no Estado do Ceará. Para tanto, foi realizada uma pesquisa exploratória, analisando o payback descontado, o valor presente líquido (VPL) e a taxa interna de retorno (TIR). Os resultados confirmaram que o projeto da instalação de energia solar fotovoltaica, além de ser uma alternativa para reduzir custos e propiciar a ampliação da matriz energética, é viável considerando os dados projetados. O retorno do investimento se dá em 6,0 anos. No final do vigésimo quinto ano o VPL será de $R \$ 1.895 .585,00$, com uma TIR de 20,29\%. A economia na geração de energia acumulada pelo sistema solar ao longo dos 25 anos será de aproximadamente $R \$ 5,3$ milhões. Tão importante quanto validar esta pesquisa, é garantir que este modelo atenderá também às demandas sociais de sustentabilidade, assunto de importância na conjectura para o desenvolvimento regional. O resultado encontrado pode ainda incentivar que as demais IES localizadas geograficamente próximas ao campus da IES em estudo adotem esta solução.
\end{abstract}

Palavras-chave: Energia solar. Instituição de Ensino Superior. Sustentabilidade. Desenvolvimento regional. Energias Renováveis.

\footnotetext{
1 Mestrando do Programa de Pós-Graduação em Desenvolvimento Regional Sustentável da Universidade Federal do Cariri - PRODER/UFCA. Engenheiro de Produção Mecânica, com especialização MBA em Gestão da Qualidade e especialização MBA em Gestão de Projetos, e-mail: allanrede@gmail.com

2 Pós-doutora em Engenharia de Produção; professora do Programa de Pós-Graduação em Desenvolvimento Regional Sustentável da Universidade Federal do Cariri (Mestrado Acadêmico), email: jeniffer.nadae@ufca.edu.br

3 Licenciado em Geografia, Mestre em Desenvolvimento Regional Sustentável e Doutor em Geografia. Professor Adjunto na Universidade Federal do Cariri (UFCA) e Coordenador do Laboratório de Estudos Urbanos, Sustentabilidade e Políticas Públicas (LAURBS), e-mail: diego.coelho@ufca.edu.br
}

R. gest. sust. ambient., Florianópolis, v. 10, n. 2, p. 84-104, jun. 2021. 


\title{
ANALYSIS OF THE ECONOMIC AND FINANCIAL FEASIBILITY PHOTOVOLTAIC SOLAR ENERGY IN A HIGHER EDUCATION INSTITUTION IN THE STATE OF CEARÁ
}

\begin{abstract}
The objective of this study is to analyze the economic and financial feasibility of installing a photovoltaic solar energy generator system in a Higher Education Institution (HEI) in the State of Ceará. For that, exploratory research was carried out, verifying the discounted payback, net present value (NPV) and internal rate of return (IRR). The results confirmed that the project for the installation of photovoltaic solar energy, in addition to being an alternative to reduce costs and provide the expansion of the energy matrix, is feasible considering the projected data. The return on investment occurs in 6.0 years. At the end of the twenty-fifth year, NPV will be $R \$$ $1,895,585.00$, with an IRR of $20.19 \%$. The savings in energy generation accumulated by the solar system over the 25 years will be approximately $R \$ 5.3$ million. As important as validating this research, it to ensuring that this model will also meet the social demands of sustainability, a matter of importance in the conjecture for regional development. The result found can also encourage other HEls located geographically close to the $\mathrm{HEl}$ campus under study to adopt this solution.
\end{abstract}

Keywords: Solar energy. Higher Education Institution. Sustainability. Regional development. Renewable energy.

\section{INTRODUÇÃO}

O desenvolvimento sustentável é considerado por diversos autores como um conceito amplo (MORIOKA et al., 2014). Segundo Seiffert (2011), ele é baseado em três dimensões, quais sejam, a o crescimento econômico, a preservação ambiental e a equidade social, em que qualquer prevalência entre uma delas provoca um desequilíbrio e deturpa o próprio conceito. Já para Sachs (2002), o desenvolvimento sustentável está pautado em 08 dimensões: ecológica, econômica, social, cultural, psicológica, territorial, política, nacional e internacional. De acordo com Waas et al. (2009), a sociedade contemporânea considera o desenvolvimento sustentável a melhor maneira de enfrentar os problemas ambientais que a humanidade enfrenta.

Segundo Tiba (2021) a energia renovável é importante para o desenvolvimento sustentável, devido à sua eficiência em atender necessidades básicas como crescimento, bem-estar humano e ambiente limpo. González et al. (2016) estudaram o uso da energia renovável no Brasil como meio de alcançar o

R. gest. sust. ambient., Florianópolis, v. 10, n. 2, p. 84-104, jun. 2021. 
desenvolvimento sustentável, e propuseram boas práticas tanto para gestores públicos quanto para a iniciativa privada.

As fontes de energia podem ser classificadas em dois tipos: fontes primárias, originadas de recursos da natureza, tais como madeira, carvão, petróleo, gás, vento, água e sol; e fontes secundárias, obtidas através da transformação das primárias, tais como eletricidade, gasolina, biomassa, energia solar e das marés (energia gravitacional) (BURGER, 2014). Além disso, a energia não se resume àquelas disponíveis nas relações comerciais ofertadas pelas indústrias de energia; existem alternativas como a solar, a eólica e a biomassa que podem ser utilizadas pela população fora das relações de mercado da indústria de energia, com aplicações específicas, simples e eficientes. Contudo, essas alternativas não integram 0 mercado de energia comercial formalmente regulado (DADALTO, 2008).

O Sol é a principal fonte de energia para os oceanos, atmosfera, solo e biosfera da Terra. Em média, durante um ano, aproximadamente 342 watts de energia solar chegam a cada metro quadrado da Terra. Esta é uma quantidade enorme de energia, 44 quadrilhões $\left(4,4 \times 10^{16}\right)$ watts de potência para ser exato. Como comparação, uma grande usina de energia elétrica produz 1 bilhão $\left(1 \times 10^{9}\right)$ de watts de potência (NASA, 2005).

O Brasil possui irradiação solar anual que varia entre 1.500 e $2.400 \mathrm{kWh} / \mathrm{m}^{2}$. Este número coloca o país em vantagem em relação aos países que são referências no uso da energia solar, como a Alemanha que possui irradiação entre 900 e 1.220 $\mathrm{kWh} / \mathrm{m}^{2}$ (BURGER, 2014). No país, as maiores incidências estão localizadas no Nordeste, região que apresenta o maior potencial solar e tendo uma estratégia de aproveitamento da geração solar consorciada com a hidrelétrica permitiria uma economia socialmente mais justa (PEREIRA, 2017). A tecnologia fotovoltaica é aquela que efetua a transformação direta da irradiação solar em energia elétrica. Possui como vantagem a possibilidade de poder ser instalada em todas as regiões do planeta, operar de forma silenciosa e possuir durabilidade de 25 anos (PHILIPPI; REIS, 2016). Ocorreu uma diminuição dos custos para a sua utilização, proporcionados pela economia de escala. Em muitos países houve incentivo governamental a fim de diversificar a matriz energética, e em linhas gerais houve aumento massivo dos sistemas conectados à rede (PHILIPPI; REIS, 2016).

R. gest. sust. ambient., Florianópolis, v. 10, n. 2, p. 84-104, jun. 2021. 
Uma das desvantagens da utilização verificada em sistemas solares fotovoltaicos de grande porte é a perda do uso do espaço, que poderia ser utilizado para outra finalidade (PHILIPPI; REIS, 2016). Entretanto, esta desvantagem pode ser facilmente mitigada, através da escolha adequada do local de instalação das placas fotovoltaicas, como, por exemplo, em telhados, lajes ou coberturas. No Brasil, a quantidade de Instituições de Ensino Superior (IES) teve um expressivo aumento nos últimos anos. Segundo o Resumo Técnico do Censo da Educação Superior 2017, o Brasil possuía 2.448 IES, enquanto em 2002 esse número era de 1.637, correspondendo a um aumento de aproximadamente 50\%. Deste total de 2.448, um número de 296 são IES públicas, o que corresponde a 12\% (BRASIL, 2019).

Diante do crescimento do número de instituições, a questão da gestão energética tem se tornado um fator importante e estratégico de governança (OYEDEPO, 2016). Como as IES são instituições dotadas de conhecimento e tecnologia, elas podem exercer diante da sociedade, a liderança perante um dos maiores desafios ambientais, construir um futuro sustentável (UHL; ANDERSON, 2001). Considerando o potencial e a vantagem ambiental e geográfica do Nordeste brasileiro, o objetivo deste estudo é analisar a viabilidade econômico-financeira da instalação de um sistema gerador de energia solar fotovoltaica de uma IES do Ceará. Para tanto, foi realizada uma pesquisa exploratória com base em dados secundários, analisando os indicadores: valor presente líquido, a taxa interna de retorno e o payback descontado, que são comumente utilizados na avaliação econômico-financeira de investimentos (GITMAN, 2014).

Este trabalho está dividido em cinco seções. A segunda seção contextualiza o tema da energia solar no Brasil; na Subseção 2.1 é explanado sobre a mini geração distribuída fotovoltaica e na Subseção 2 são apresentados os indicadores econômico-financeiros para embasar a análise de viabilidade. Na Seção 3 é apresentada a metodologia, na Seção 4 são apresentados os resultados e discussão, e na Seção 5 as considerações finais.

\section{FUNDAMENTAÇÃO TEÓRICA}

Nesta seção serão apresentados os fundamentos teóricos, que aqui pretendem embasar as análises do presente trabalho.

R. gest. sust. ambient., Florianópolis, v. 10, n. 2, p. 84-104, jun. 2021. 


\subsection{Uso de energia solar no Brasil}

A energia solar é aquela gerada diretamente pelo Sol e consiste na transformação da energia radiante do sol em energia elétrica por meio de células fotovoltaicas (BURGER, 2014). Com relação a este tipo de energia, percebe-se um grande potencial a ser explorado tanto pela sua relevante praticidade em utilização quanto pelo aproveitamento eficaz dessa forma de energia.

Conforme Silva (2015), quase todas as fontes de energia são formas indiretas de energia solar: energia hidráulica, biomassa, eólica, combustíveis fósseis e energia dos oceanos. Ainda segundo o mesmo autor, de forma direta, a radiação solar pode ser convertida diretamente em energia elétrica, por meio de efeito fotovoltaico. Assim, a energia liberada pelo sol pode ser aproveitada através da devida coleta e conversão em energia elétrica de maneira prática e sustentável.

Na Tabela 1, observa-se que o Balanço Energético Nacional - BEN 2019 (ano base 2018) informa que, no Brasil, a capacidade instalada de energia elétrica é de 162.840 MW (megawatt), dos quais 1.798 MW provêm da energia solar. Já no Nordeste (NE), a capacidade instalada é de $35.063 \mathrm{MW}$, dos quais 1.246 MW provêm da energia solar. Dentre os estados do NE, o Ceará apresenta uma capacidade instalada de energia de 4.364 MW, dos quais $137 \mathrm{MW}$ provêm da energia solar. Diante disso, percebe-se que 3,13\% da energia utilizada no Ceará é proveniente da energia solar, o que demonstra o quanto este tipo de energia deve ser incentivado, a fim de propiciar o desenvolvimento sustentável regional. Uma economia solar promove a descentralização da geração de energia, o que permitiria o crescente controle comunitário do sistema energético (ACOSTA, 2016).

Tabela 1: Capacidade Instalada de Geração Elétrica

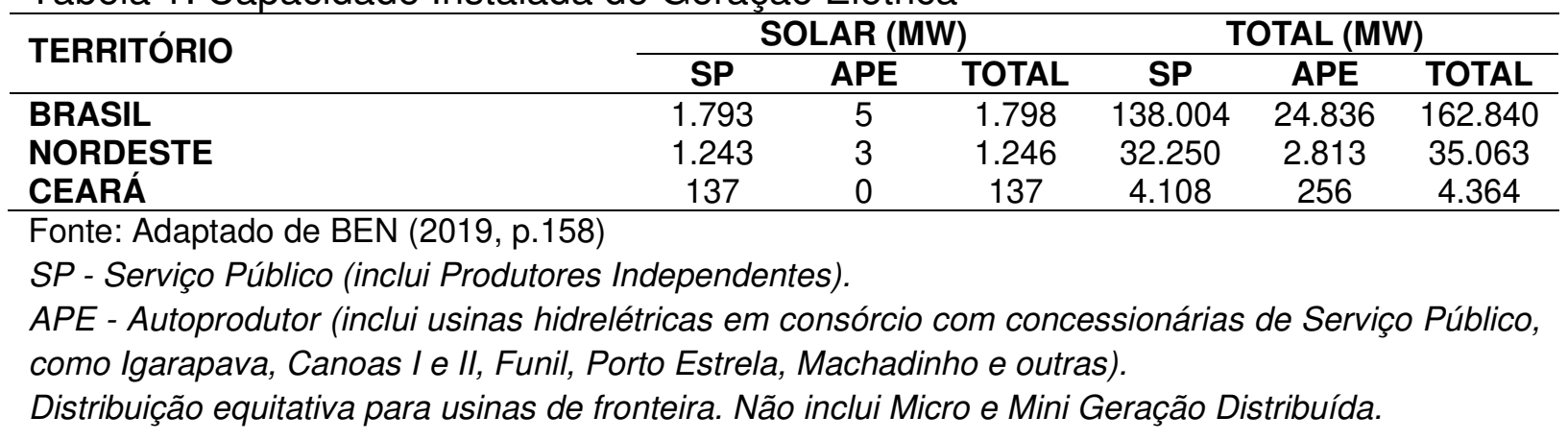

Segundo o BEN 2019, o consumo final de energia no Brasil foi de 535.403 GWh (gigawatt-hora), sendo que o setor público consumiu 44.126 GWh, R. gest. sust. ambient., Florianópolis, v. 10, n. 2, p. 84-104, jun. 2021. 
representando $8,24 \%$ do total. A oferta interna de energia no mesmo período foi de 636.375 GWh. Embora o Brasil tenha consumido 84,13\% da energia ofertada, não é necessário um colapso no fornecimento de energia elétrica para que se tomem ações sustentáveis. Para efeito comparativo, o aumento no consumo de energia elétrica em relação ao ano anterior foi de 1,4\%, excedendo a taxa de crescimento demográfico, que foi de $0,99 \%$ (Tabela 2), revelando que são os hábitos que estão causando este aumento no consumo.

Tabela 2: Oferta Interna de Energia / População

\begin{tabular}{llrr}
\hline & Unidade & $\mathbf{2 0 1 7}$ & $\mathbf{2 0 1 8}$ \\
\hline Oferta Interna de Energia Elétrica - OIEE & GWh & 625.682 & 636.375 \\
População' Residente - POP & $10^{6}$ hab & 207,6 & 209,3 \\
\hline Fonte: Adaptado de BEN (2019, p. 136) & & \\
1 Estimativa elaborada pela Empresa de Pesquisa Energética baseada na 'Projeção da População do \\
Brasil por sexo e idade: 2000-2060' - agosto de 2013 (IBGE)
\end{tabular}

\subsection{Micro e Minigeração Distribuída de energia}

A micro e a minigeração distribuída de energia tem se mostrado uma alternativa para a diversificação da matriz energética, que segundo a Agência Nacional de Energia Elétrica (ANEEL) é definida como (2016, p.11):

A micro e a minigeração distribuída consistem na produção de energia elétrica a partir de pequenas centrais geradoras que utilizam fontes renováveis de energia elétrica ou cogeração qualificada, conectadas à rede de distribuição por meio de instalações de unidades consumidoras.

(...) a microgeração distribuída refere-se a uma central geradora de energia elétrica, com potência instalada menor ou igual a 75 quilowatts (kW), enquanto que a minigeração distribuída diz respeito às centrais geradoras com potência instalada superior a $75 \mathrm{~kW}$ e menor ou igual a 3 megawatt (MW), para a fonte hídrica, ou $5 \mathrm{MW}$ para as demais fontes.

Portanto, o que diferencia a micro da minigeração distribuída, é que esta possui uma potência maior em relação àquela, sendo a cogeração qualificada definida como processo de geração de energia elétrica combinada com 0 aproveitamento da energia térmica dissipada pelo gerador, atendendo a requisitos de eficiência e potência específicos (ANEEL, 2006). A implantação de uma central de minigeração distribuída pode proporcionar vantagens, dentre elas, estão a eletricidade gerada a um menor custo para o consumidor e a diminuição do uso de fontes de energia não renováveis (BARBOSA, 2013). Uma opção para reduzir os gastos públicos com energia elétrica é a instalação de uma central de minigeração

R. gest. sust. ambient., Florianópolis, v. 10, n. 2, p. 84-104, jun. 2021. 
distribuída de energia, pois o setor vem sofrendo com o aumento da tarifa energética nos últimos anos, devido à crise hídrica que o país vem passando. Segundo Hobmeir e Trindade (2016), este aumento foi de mais de 35\%, além da introdução do sistema de bandeiras tarifárias, amarelas e vermelhas. Assim, a ANEEL, em sua Resolução 482, que passou a vigorar em 2012, permitiu ao consumidor gerar sua própria energia elétrica, de maneira a ser injetada na rede elétrica existente, com o objetivo de reduzir o gasto com energia elétrica vinda da concessionária. Para o escopo do Estudo Brasil, a tarifa de energia elétrica consiste num valor único $(\mathrm{R} \$ / \mathrm{kWh}$ ) aplicado ao consumo de eletricidade mensal $(\mathrm{kWh})$ e sobre o qual incidem impostos federais (PIS e COFINS) e estaduais (ICMS), sendo este último variável de acordo com o Estado, tipo de cliente e consumo (LANDEIRA, 2013). Na Figura 1 está ilustrado o sistema fotovoltaico, sendo o Grupo A os consumidores conectados em alta tensão e o Grupo B os conectados em baixa tensão.

Figura 1: Sistema de compensação de Energia Elétrica. Sistema fotovoltaico conectado à rede

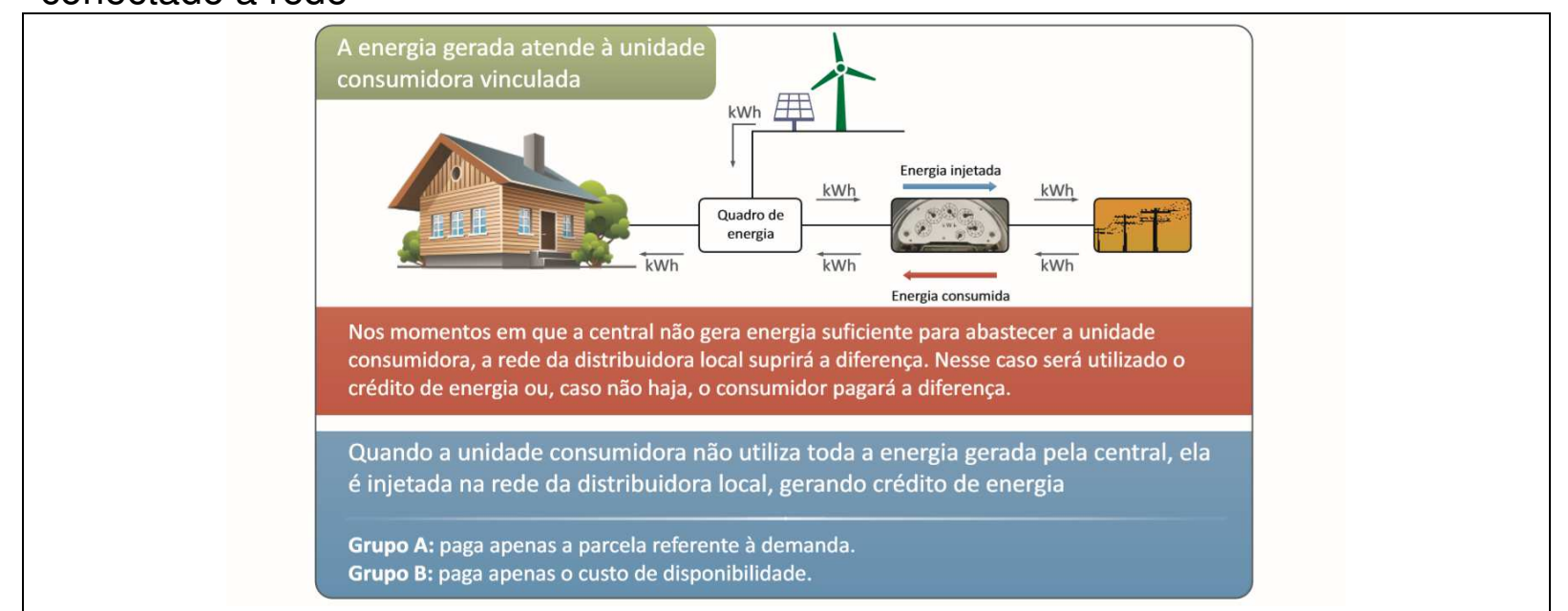

Fonte: Cadernos temáticos ANEEL (2016, p. 16)

As vantagens de se produzir sua própria energia são inúmeras. Para Guangul (2019), a energia fornecida pelo sol é uma das principais fontes de energia ilimitada disponível na Terra, sendo uma energia ecologicamente amigável, por ser não poluente. Outras vantagens estão no fato de possuírem fácil instalação e manutenção. Uma das desvantagens em utilizar esta tecnologia é que ela não gera energia durante a noite, e perde eficiência em dias nublado ou chuvoso (MACHADO; MIRANDA, 2015). A eficiência da conversão da energia solar em energia elétrica é R. gest. sust. ambient., Florianópolis, v. 10, n. 2, p. 84-104, jun. 2021. 
considerada baixa, não excedendo $20 \%$ para sistemas domésticos e industriais, o que acaba por demandar um maior espaço para instalar mais painéis solares.

\subsection{Indicadores para análise de viabilidade econômico-financeira de projetos}

Os indicadores financeiros para justificar a instalação de um sistema de geração de energia solar variam de acordo com cada projeto. Para determinar a viabilidade econômico-financeira, comumente são utilizados a Taxa Interna de Retorno (TIR), o Valor Presente Líquido (VPL) e o payback descontado (Quadro 1), podendo ser utilizado outros indicadores para complementar a análise, se necessário.

Quadro 1: Indicadores de análise de viabilidade econômico-financeira

\begin{tabular}{|l|l|}
\hline $\begin{array}{l}\text { Indicador } \\
\text { (VPL) }\end{array}$ & $\begin{array}{l}\text { Definição } \\
\text { V soma algébrica dos valores descontados do fluxo de caixa a ele } \\
\text { associados. Em outras palavras, é a diferença do valor presente das } \\
\text { receitas menos o valor presente dos custos (a). Sua fórmula é dada por: }\end{array}$ \\
$\qquad \sum_{t=1}^{n} \frac{F C_{t}}{(1+k)^{t}}-F C_{0}$ \\
\hline $\begin{array}{l}\text { Taxa interna de retorno } \\
\text { (TIR) }\end{array}$ & $\begin{array}{l}\text { Sendo } F C_{0} \text { o investimento inicial do projeto, FCt o valor presente de suas } \\
\text { entradas de caixa, } t \text { o momento em que o fluxo de caixa ocorreu; } k=\text { taxa } \\
\text { É a taxa de desconto que iguala o VPL de uma oportunidade de } \\
\text { investimento a zero (GITMAN, 2004). Pode ser entendida como a taxa de } \\
\text { juros em um investimento que anula o VPL do fluxo de caixa. Utiliza a } \\
\text { mesma fórmula acima, igualando o VPL a zero. }\end{array}$ \\
\hline Payback descontado & $\begin{array}{l}\text { O payback corresponde ao período de tempo necessário para que as } \\
\text { entradas de caixa se igualem ao valor a ser investido, ou seja, o prazo de } \\
\text { recuperação de um investimento. Em projetos, ele pode estabelecer um } \\
\text { período máximo para o retorno do projeto, procurando-se reduzir o risco. } \\
\text { (LEMESJÚNIOR; RIGO; CHEROBIM, 2002). }\end{array}$ \\
\hline
\end{tabular}

Fonte: Elaborado pelo autor (2020).

\subsubsection{Valor Presente Líquido}

Ao utilizar o VPL para tomar decisões, quando o VPL for maior que zero, o projeto é financeiramente viável. Quando o VPL for menor que zero, o projeto não é viável. Assim, "a análise do valor presente líquido pode ser usada para avaliar e escolher - do ponto de vista financeiro - a melhor proposta de investimento para uma organização" (CERTO; PETER, 2005, p. 232). O Valor Presente Líquido (VPL) leva explicitamente em conta o valor do dinheiro no tempo, é considerado uma técnica R. gest. sust. ambient., Florianópolis, v. 10, n. 2, p. 84-104, jun. 2021. 
sofisticada de orçamento de capital (GITMAN, 2004), e geralmente é o índice de avaliação econômica dinâmica mais utilizado (ZHANG et al., 2021). Portanto, o VPL busca trazer para o presente os valores dos fluxos de caixa ao longo do tempo e tem sido utilizado amplamente na área financeira. Desta maneira, quanto maior for o VPL de um projeto, maior será a atratividade do mesmo.

\subsubsection{Taxa interna de retorno}

Considerando os valores de caixa ocorrendo em diferentes momentos, conclui-se que o método da TIR, ao levar em conta o dinheiro no tempo, expressa, a rentabilidade, em caso de aplicação, ou custo, no caso de um financiamento do fluxo de caixa (ASSAF NETO, 2007). Quanto maior for a TIR de um projeto, maior será a atratividade do mesmo.

\subsubsection{Payback descontado}

O payback descontado é semelhante ao payback, sendo que naquele calculase o tempo de retorno do capital investido a partir do valor presente dos fluxos de caixa, considerando o custo de capital (FONSECA; BRUNI, 2003). No payback descontado o investidor determina a taxa mínima de atratividade (TMA), que serve como comparativo para a remuneração do capital investido (LEMES JÚNIOR; RIGO; CHEROBIM, 2002). Portanto, quanto menor for o payback descontado de um projeto, maior será a atratividade do mesmo. A importância de utilizar estes indicadores é que, segundo Castanheira (2016, p.145), "sempre que precisamos tomar uma decisão de investir ou não em algum empreendimento, a TIR e o VPL são ferramentas que nos ajudam a seguir o caminho mais acertado."

\section{METODOLOGIA}

Neste estudo foi realizada uma pesquisa exploratória, visto que teve como objetivo a busca de informações sobre o assunto em uma IES no Estado do Ceará. Os dados secundários foram coletados na própria IES, através da análise do histórico da conta de energia, da análise da estrutura física predial e do banco de dados do CRESESB (2020). A pesquisa exploratória é aquela que tem como principal característica a informalidade, a flexibilidade e a criatividade (SAMARA; BARROS, 2007), e é ainda realizado a partir de dados secundários, aqueles já

R. gest. sust. ambient., Florianópolis, v. 10, n. 2, p. 84-104, jun. 2021. 
disponíveis. Segundo Melo (2020) para se dimensionar um sistema fotovoltaico, deve-se avaliar o consumo de energia elétrica nos últimos dozes meses do local em que será instalado o sistema. Para Elisardo e Wippel (2018) é realizada a média aritmética de consumo energético dos últimos doze meses de faturamento para se calcular a capacidade do sistema fotovoltaico. Portanto, os dados obtidos na própria IES são referentes às contas de energia no período de abril de 2019 a março de 2020. Em seguida foi realizado estudo sobre a parte da estrutura predial onde se pretende realizar a instalação do sistema gerador de energia solar fotovoltaica.

O banco de dados do CRESESB forneceu importantes informações referentes à irradiação solar, tendo em vista que estão diretamente relacionados a eficiência do sistema com a localização geográfica. Através do programa SunData, há o cálculo da irradiação solar diária mensal em qualquer ponto do território nacional (CRESESB, 2020). Com o lançamento das coordenadas geográficas, latitude e longitude, o programa fornece os dados contendo o valor da irradiação solar diária média mensal em $\mathrm{kWh} / \mathrm{m}^{2}$.dia, para todos os meses do ano. Para efeito comparativo, o programa fornece esta informação considerando quatro planos de inclinação, para que se faça a escolha do melhor rendimento possível para o sistema: plano horizontal com inclinação $0^{\circ} \mathrm{N}$; ângulo igual a latitude; ângulo de inclinação para fornecer a maior média anual; ângulo de inclinação para obter o maior mínimo mensal. Desde 2017, o CRESESB obteve autorização para utilizar o Atlas Brasileiro de Energia Solar para atualizar a base de dados do SunData. De posse dos dados levantados, foi realizada análise técnica de dimensionamento do sistema para identificar a capacidade de geração do sistema proposto, bem como identificar o percentual de atendimento da demanda atual. Esta análise considerou a quantidade de painéis solares e demais componentes, com descrição técnica, junto com o orçamento dos equipamentos e orçamento para uma possível instalação. E foram feitas as análises de viabilidade econômico-financeira.

\section{RESULTADOS E DISCUSSÃO}

Neste tópico será abordada uma contextualização breve sobre a IES, bem como um descritivo do consumo considerado para a realização deste trabalho. $\mathrm{Na}$ sequência serão apresentados os resultados.

R. gest. sust. ambient., Florianópolis, v. 10, n. 2, p. 84-104, jun. 2021. 


\subsection{Caracterização da Instituição de Ensino Superior}

A pesquisa foi realizada em uma IES localizada no Estado do Ceará, distribuída em 05 campi, a IES possui 21 cursos de graduação e 15 cursos de pósgraduação, sendo quatro mestrados e um doutorado. De acordo com o relatório consolidado da instituição de 2018, a IES possui um quantitativo de 2.847 alunos. $O$ campus escolhido para a pesquisa fica localizado em Crato-CE, município que faz parte da Região Metropolitana do Cariri (RMC). Por sua vez, a RMC está inserida na região semiárida do nordeste brasileiro, e é constituída por nove municípios, sendo eles: Barbalha, Caririaçu, Crato, Farias Brito, Jardim, Juazeiro do Norte, Milagres, Nova Olinda e Santana do Cariri (LIMA et al. 2021). O mencionado campus foi inaugurado em 2011 e conta com dois cursos de graduação e um de mestrado em Desenvolvimento Regional Sustentável, um dos primeiros do país ligado à temática da sustentabilidade, funcionando em horário diurno. Suas instalações possuem $4.668 \mathrm{~m}^{2}$ de área construída e 10 hectares disponíveis para as atividades de campo, cultivo experimentais e criações. O estudo foi feito com base na instalação de um sistema Gerador Fotovoltaico conectado à rede (conhecido como sistema on-grid ou grid-tie). Atualmente a energia consumida pela IES é proveniente da concessionária de energia elétrica do estado. A análise da fatura de energia elétrica da IES nos permite verificar que há consumo do horário ponta e no horário fora ponta, sendo o valor médio do $\mathrm{kWh}$ de $\mathrm{R} \$ 2,0586$ e $\mathrm{R} \$ 0,4382$, respectivamente.

Percebe-se que o consumo do kWh no horário ponta é mais caro do que o consumo do kWh no horário fora ponta (aproximadamente 4,76 vezes mais caro). Significa dizer que o sistema fotovoltaico precisa gerar 4,76 kWh para compensar cada 1 kWh consumido no horário ponta. Como a geração de energia solar fotovoltaica se dará no horário fora ponta, que é o horário em que há geração de energia por parte do sol, cada $1 \mathrm{kWh}$ gerado pelo sistema compensará o equivalente a $1 \mathrm{kWh}$ consumido no horário fora ponta, ao custo de $\mathrm{R} \$ 0,4382$ por cada kWh. Desta maneira, na Tabela 3 está apresentado o consumo de energia da IES, no período de abril de 2019 a março de 2020, referente ao horário fora ponta.

Tabela 3: Consumo mensal fora ponta, histórico de 12 meses

Mês de referência $\mathrm{mar} / 20$

$\mathrm{fev} / 20$

jan/20

$\mathrm{dez} / 19$

\section{Consumo fora ponta (kWh)}

14.740

14.403

24.811

14.665

R. gest. sust. ambient., Florianópolis, v. 10, n. 2, p. 84-104, jun. 2021. 


$\begin{array}{cr}\text { nov/19 } & 21.564 \\ \text { out/19 } & 18.084 \\ \text { set/19 } & 16.534 \\ \text { ago/19 } & 12.215 \\ \text { jul/19 } & 14.605 \\ \text { jun/19 } & 15.858 \\ \text { mai/19 } & 13.298 \\ \text { abr/19 } & 15.817 \\ \text { Média } & 16.383\end{array}$

Fonte: Dados da pesquisa.

Portanto, neste estudo será considerada a geração pelo sistema solar fotovoltaico equivalente ao consumo do horário fora ponta, devido ao custo menor do kWh. A média mensal de consumo neste período foi de $16.383 \mathrm{kWh}$, e que, portanto, embasou a presente análise econômico-financeira.

\subsection{Proposta de instalação do sistema fotovoltaico}

O sistema fotovoltaico poderá ser conectado em paralelo à rede elétrica que existe na universidade, não havendo a necessidade de instalação de banco de baterias e, caso haja excedente de geração, este será fornecido para a rede de distribuição local. Para dimensionar adequadamente um sistema fotovoltaico conectado à rede elétrica, é preciso quantificar a média de horas de sol por dia no local da instalação, pois esta informação impacta diretamente no rendimento dos módulos solares. Os dados apresentados neste estudo estão disponibilizados no programa SunData, fornecido pelo CRESESB, que calcula a irradiação solar diária mensal no território nacional para qualquer ponto de referência através das coordenadas de latitude e longitude. Desta maneira, foram utilizadas as coordenadas contidas na Figura 2 e obtidos os seguintes dados abaixo, que representam a variação da irradiação global diária média mensal que incide na superfície, comparada com inclinações variadas.

Figura 2: Plano inclinado: Cálculo irradiação solar diária média mensal [kWh/m².dia]

\begin{tabular}{|c|c|c|c|c|c|c|c|c|c|c|c|c|c|c|c|c|c|}
\hline \multicolumn{18}{|c|}{ 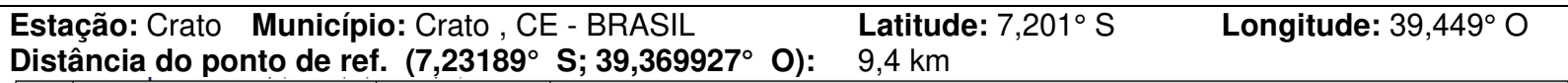 } \\
\hline \multirow{2}{*}{ \# } & \multirow{2}{*}{ Ângulo } & \multirow{2}{*}{ Inclinação } & \multicolumn{15}{|c|}{ Irradiação solar diária média mensal [kWh $/ \mathrm{m}^{2}$.dia] } \\
\hline & & & $\operatorname{Jan}$ & \begin{tabular}{|l|l} 
Fev & i \\
\end{tabular} & \begin{tabular}{l|l} 
Mar & $\mathrm{A}$ \\
\end{tabular} & \begin{tabular}{l|l} 
br & $M$ \\
\end{tabular} & & Jun & \begin{tabular}{l|l} 
Jul & $A$ \\
un
\end{tabular} & \begin{tabular}{|l|l} 
Ago & $S$ \\
\end{tabular} & iet & out & \begin{tabular}{l|l} 
Nov & t \\
\end{tabular} & Dez & Média & Delta & \\
\hline$\square$ & Plano Horizontal & $0^{\circ} \mathrm{N}$ & 5,83 & \begin{tabular}{|l|}
5,76 \\
\end{tabular} & \begin{tabular}{|l|r|}
6,568 \\
\end{tabular} & 5,46 & 5,15 & 4,97 & 5,33 & \begin{tabular}{|l|}
6,12 \\
\end{tabular} & 6,59 & \begin{tabular}{l|l|}
9 & 6,56 \\
\end{tabular} & 6,55 & \begin{tabular}{|l|l|}
5 & 6,19 \\
\end{tabular} & \begin{tabular}{l|l}
19 & 5,8 \\
\end{tabular} & $\begin{array}{ll}85 & 1 \\
\end{array}$ & 1,61 \\
\hline$\nabla$ & Ângulo igual a latitude & $7^{\circ} \mathrm{N}$ & 5,59 & 5,62 & 5,67 & 5,61 & 5,42 & 5,30 & 5,66 & 6,38 & 6,66 & 6,44 & 6,28 & 5,89 & $5,8 \mathrm{C}$ & 88 & 1,36 \\
\hline$\nabla$ & Maior média anual & $7^{\circ} \mathrm{N}$ & 5,59 & 5,62 & 5,67 & 5,61 & 5,42 & 5,30 & 5,66 & 6,38 & 6,66 & 6,44 & 6,28 & 5,89 & $5,8 \mathrm{C}$ & 88 & 1,36 \\
\hline 可 & Maior mínimo mensal & $10^{\circ} \mathrm{N}$ & 5,46 & 5,55 & \begin{tabular}{l|l}
5,65 \\
\end{tabular} & 5,65 & 5,51 & 5,43 & 5,78 & 6,46 & $\begin{array}{ll}6,67 \\
\end{array}$ & 6,37 & 6,15 & 5,75 & 5,8 & 87 & 1,24 \\
\hline
\end{tabular}

Fonte: CRESESB (2020, online).

R. gest. sust. ambient., Florianópolis, v. 10, n. 2, p. 84-104, jun. 2021. 
O CRESESB apresenta os dados da irradiação solar diária média mensal para o plano horizontal, ao ângulo igual à latitude, de maior média e de maior mínimo. Esta informação orienta a escolha do sistema mais eficiente, pois a inclinação está diretamente relacionada à captação da irradiação solar. Para este projeto, foi considerada a inclinação de 7 graus, que apresenta o maior valor na coluna média, e que permitirá dimensionar a quantidade de painéis e a potência do inversor. Além do ganho de eficiência, a importância de utilizar um ângulo de inclinação na instalação dos painéis se justifica pelo fato de que a instalação no plano horizontal favorece o acúmulo de sujeira, o que pode gerar interferência na geração e aumento nos custos de manutenção. Portanto, para uma inclinação de 7 graus, a média anual de irradiação é de $5,88 \mathrm{kWh} / \mathrm{m}^{2}$.dia, com geração máxima no mês de setembro e geração mínima no mês de junho com 6,66 kWh/m².dia e $5,30 \mathrm{kWh} / \mathrm{m}^{2}$.dia, respectivamente, (Figura 3). Estas informações serão adotadas para realizar a próxima etapa, que consiste no dimensionamento do sistema proposto.

Figura 3: representação gráfica, irradiação solar diária média mensal [kWh/m².dia]

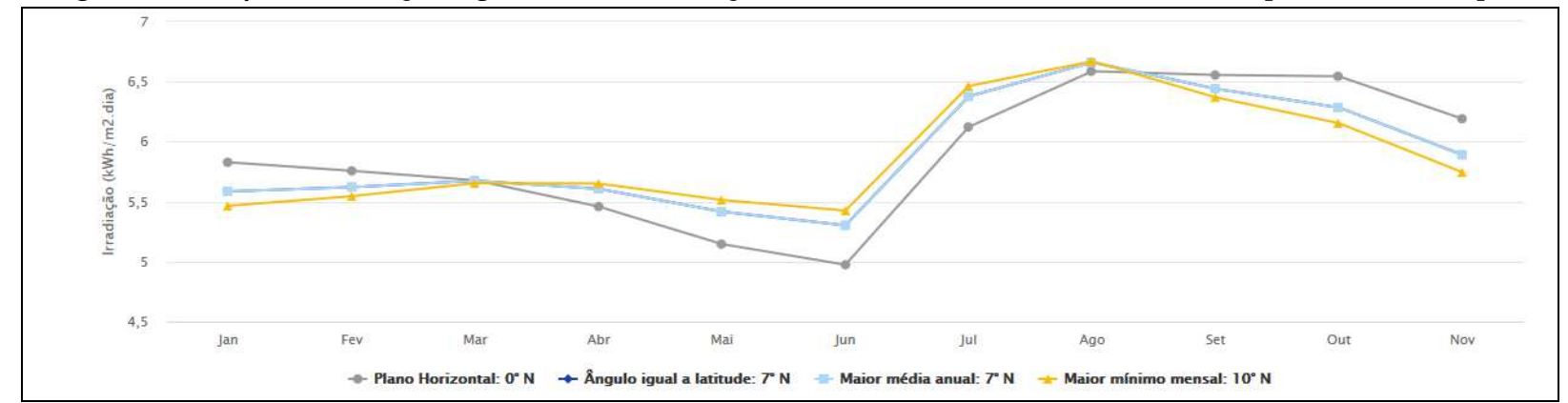

Fonte: CRESESB (2020, online).

\subsection{Dimensionamento e investimento do sistema proposto}

De posse do histórico de consumo da IES em estudo, juntamente com os dados coletados, entrou-se em contato com uma empresa local que fornece soluções para sistemas de energia solar fotovoltaica, para que fosse elaborada uma proposta formal dos custos contendo as informações técnicas para a instalação do sistema fotovoltaico que atendesse à demanda energética da IES. Na Tabela 4 está apresentada a composição do sistema proposto, referente à descrição dos equipamentos, do custo unitário e do investimento total necessário. Cabe destacar que a proposta não cobre reforços e adaptações na rede elétrica interna (imóvel)

R. gest. sust. ambient., Florianópolis, v. 10, n. 2, p. 84-104, jun. 2021. 
e/ou externa; e não abrange alterações estruturais ou reformas no prédio. Estas, caso fossem necessárias, deveriam ser providenciadas pelo cliente.

A energia elétrica que será gerada pelo sistema fotovoltaico proposto supre uma demanda de aproximadamente $100 \%$ do consumo fora ponta de energia elétrica do campus, de acordo com a análise do histórico de consumo de energia apresentado na Tabela 3. A instalação dos painéis solares relacionados será feita na parte superior da estrutura predial, sobre a laje da edificação, em suportes de sustentação de aço.

Tabela 4: Detalhamento dos equipamentos e orçamento do sistema proposto

\begin{tabular}{lrrr}
\hline Descrição do material & Qtd. & Valor R\$(unt) & Valor R\$(total) \\
\hline PainelLongi 144Cells 385W Half Mono Perc & 324 & $1.270,89$ & $411.768,36$ \\
Inversor ABB PVS50-TL-SX2 & 2 & $51.470,97$ & $102.941,94$ \\
Estrutura de Fixação & 1 & $20.588,39$ & $20.588,39$ \\
Projeto Elétrico & 1 & $23.133,02$ & $23.133,02$ \\
Comissionamento e start-up & 1 & $3.500,00$ & $3.500,00$ \\
Instalações elétricas das placas fotovoltaicas & 1 & $4.000,00$ & $4.000,00$ \\
Painel de proteção e distribuição de ener. fotov. (PPDEF) & 1 & $6.000,00$ & $6.000,00$ \\
Interligação com a rede de energia existente & 1 & $3.000,00$ & $3.000,00$ \\
Total geral & & & $\mathbf{5 7 4 . 9 3 1 , 7 1}$ \\
\hline
\end{tabular}

Fonte: Dados da pesquisa (2020)

Os painéis elétricos e os inversores serão alocados e instalados em uma sala adequada, na própria edificação, de acesso restrito. A localização será escolhida a fim de facilitar a manutenção, realizar o monitoramento da geração de energia bem como gerenciar a eficiência do sistema. Toda a parte referida à instalação, que abrange desde a elaboração do projeto elétrico, comissionamento e start-up, instalações elétricas das placas fotovoltaicas, instalação da proteção do sistema e interligação com a rede elétrica da concessionária, será realizada por empresa devidamente capacitada, especialista no assunto, e com experiência comprovada na área de energia solar fotovoltaica. De acordo com as exigências legais vigente, o projeto elétrico é encaminhado à concessionária de energia elétrica, para que esta possa emitir o parecer de acesso, realizar a vistoria, regularizar os aspectos técnicos, aprovar o ponto, trocar medição e iniciar o sistema de compensação. $O$ objetivo destes procedimentos é garantir que a instalação será realizada obedecendo aos padrões de segurança e funcionará corretamente. Para este sistema, o investimento é de $\mathrm{R} \$ 574.931,71$ conforme exposto na Tabela 3.

R. gest. sust. ambient., Florianópolis, v. 10, n. 2, p. 84-104, jun. 2021. 


\subsection{Análise da viabilidade econômico-financeira}

O sistema proposto será constituído de 324 painéis fotovoltaicos de $385 \mathrm{Wp}$ cada, possuindo o sistema, portanto, potência pico de 124,74kW, sendo classificado como minigeração distribuída. Na Figura 4 está apresentada a energia que será fornecida pelo sistema, mês a mês, e a média do período, podendo-se constatar que a partir do mês de agosto a energia gerada é maior, devido à maior irradiação solar neste período do ano para a localização geográfica determinada.

$\mathrm{Na}$ Tabela 5 está apresentado o fluxo de caixa projetado para o período de 25 anos. Foi considerado um reajuste tarifário de energia de $7 \%$ a.a., conforme utilizado por Conti (2021).

Figura 4: Energia média gerada pelo sistema em $\mathrm{kWh} / \mathrm{mês}$

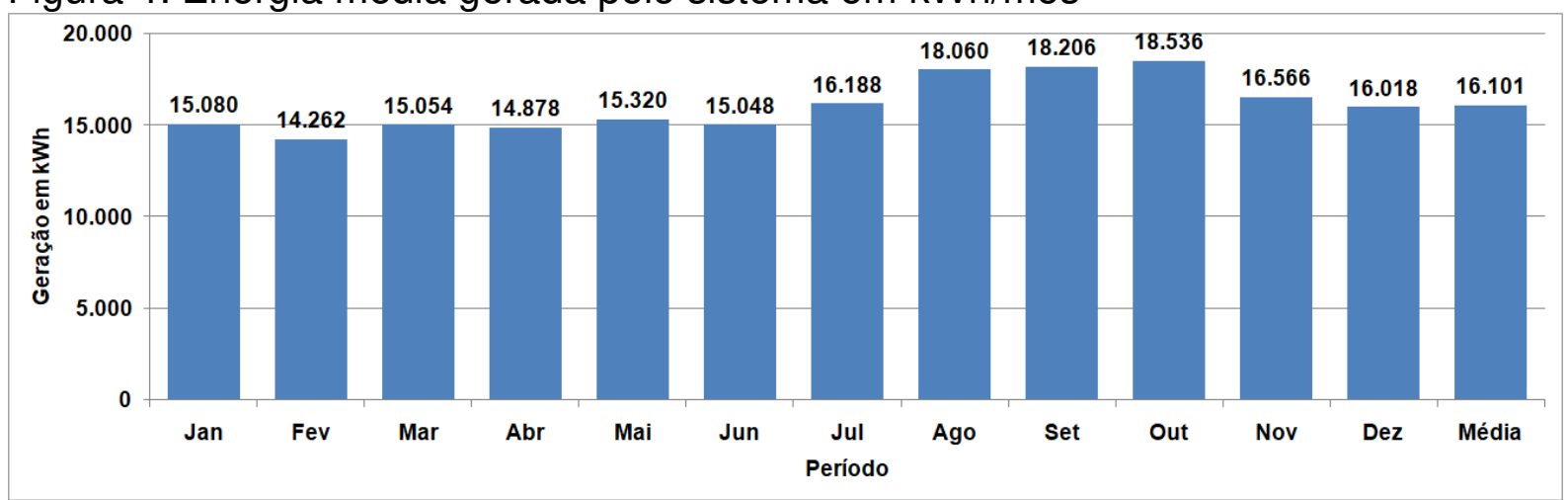

Fonte: Dados do Autor.

Para se determinar a TMA, muitos autores optaram por utilizar o valor da taxa Sistema Especial de Liquidação e de Custódia (SELIC). Souza e da Penha (2020) e Magnus (2019) utilizaram em seus cálculos a SELIC de 5,00\%, referente ao período de outubro de 2019; Albuquerque (2020) considerou em seu estudo a SELIC a 2,25\%, referente a junho de 2020; Reis (2020) adotou o valor da SELIC a 2,00\%, referente ao período de dezembro de 2020. A taxa SELIC corresponde à taxa básica de juros da economia e influencia todas as taxas de juros do país, tais quais as dos empréstimos e financiamentos (BANCO CENTRAL, 2021a), sendo, portanto, considerada como taxa mínima de atratividade em diversos cálculos de financiamento. O Comitê de Política Monetária (COPOM) fixou em março de 2021 a taxa SELIC para 2,75\% (BANCO CENTRAL, 2021b). Entretanto, Fernandes, Silva e Belchior (2020), consideraram em seu estudo uma taxa superior à da SELIC, com o objetivo de agregar conservadorismo ao projeto, visto que os recursos financeiros para a instalação do sistema solar fotovoltaico são provenientes dos cofres públicos.

R. gest. sust. ambient., Florianópolis, v. 10, n. 2, p. 84-104, jun. 2021. 
Portanto, por conservadorismo, para este estudo foi considerada uma TMA de $5,00 \%$, valor superior à taxa SELIC fixada em $2,75 \%$. A taxa de manutenção e operação fixa anual foi estimada em $1 \%$ do valor inicial do sistema, de acordo com Nakabayashi (2014). Os dados para a análise de viabilidade econômico-financeira são apresentados na Tabela 5.

Considerando o custo médio do $\mathrm{kWh}$ de $\mathrm{R} \$ 0,4382$ e o sistema proposto com capacidade de geração média mensal de 16.101 kWh é possível economizar no primeiro ano $R \$ 84.665,50$, com uma média mensal de $R \$ 7.055,46$. O cálculo do payback descontado considerou o fluxo de caixa acumulado, sendo necessários 6,0 anos para recuperar o investimento inicial do sistema proposto.

Tabela 5: Fluxo de caixa projetado e Valor Presente Líquido (VPL)

\begin{tabular}{|c|c|c|c|c|c|c|c|}
\hline Ano & Investimento & $\begin{array}{c}\text { Economia } \\
\text { na } \\
\text { geração } \\
\text { de energia }\end{array}$ & $\begin{array}{l}\text { Operação e } \\
\text { manutenção }\end{array}$ & $\begin{array}{l}\text { Fluxo de } \\
\text { caixa }\end{array}$ & $\begin{array}{c}\text { Fluxo de } \\
\text { caixa } \\
\text { acumulado }\end{array}$ & $\begin{array}{c}\text { Fluxo de } \\
\text { caixa } \\
\text { descontado }\end{array}$ & $\begin{array}{c}\text { Payback } \\
\text { descontado }\end{array}$ \\
\hline 0 & $-\mathrm{R} \$ 574.932$ & $\mathrm{R} \$ 0$ & $\mathrm{R} \$ 0$ & $-\mathrm{R} \$ 574.932$ & $-\mathrm{R} \$ 574.932$ & $-\mathrm{R} \$ 574.932$ & $-\mathrm{R} \$ 574.932$ \\
\hline 1 & $R \$ 0$ & $\mathrm{R} \$ 84.665$ & $-R \$ 5.749$ & $\mathrm{R} \$ 78.916$ & $-R \$ 496.016$ & & $-R \$ 499.773$ \\
\hline 2 & $\mathrm{R} \$ 0$ & $\mathrm{R} \$ 90.592$ & $-\mathrm{R} \$ 5.749$ & $\mathrm{R} \$ 84.843$ & $-\mathrm{R} \$ 411.173$ & $\mathrm{R} \$ 76.955$ & $-R \$ 422.819$ \\
\hline 3 & $\mathrm{R} \$ 0$ & $\mathrm{R} \$ 96.934$ & $-R \$ 5.749$ & $\mathrm{R} \$ 91.184$ & $-R \$ 319.989$ & $\mathrm{R} \$ 78.768$ & $-R \$ 344.050$ \\
\hline 4 & $\mathrm{R} \$ 0$ & $\mathrm{R} \$ 103.719$ & $-\mathrm{R} \$ 5.749$ & $\mathrm{R} \$ 97.970$ & $-\mathrm{R} \$ 222.019$ & $\mathrm{R} \$ 80.600$ & $-R \$ 263.450$ \\
\hline 5 & $\mathrm{R} \$ 0$ & $\mathrm{R} \$ 110.979$ & $-R \$ 5.749$ & $R \$ 105.230$ & $-R \$ 116.789$ & $\mathrm{R} \$ 82.450$ & $-R \$ 181.000$ \\
\hline 6 & $\mathrm{R} \$ 0$ & $R \$ 118.748$ & $-\mathrm{R} \$ 5.749$ & $R \$ 112.998$ & $-R \$ 3.791$ & $\mathrm{R} \$ 84.321$ & $-R \$ 96.679$ \\
\hline 7 & $\mathrm{R} \$ 0$ & $\mathrm{R} \$ 127.060$ & $-R \$ 5.749$ & $\mathrm{R} \$ 121.311$ & $\mathrm{R} \$ 117.520$ & $\mathrm{R} \$ 86.213$ & $-R \$ 10.466$ \\
\hline 8 & $\mathrm{R} \$ 0$ & $R \$ 135.954$ & $-R \$ 5.749$ & $R \$ 130.205$ & $R \$ 247.725$ & 88.128 & $\mathrm{R} \$ 77.662$ \\
\hline 9 & $\mathrm{R} \$ 0$ & $\mathrm{R} \$ 145.471$ & $-R \$ 5.749$ & $\mathrm{R} \$ 139.722$ & $\mathrm{R} \$ 387.447$ & $\mathrm{R} \$ 90.066$ & $\mathrm{R} \$ 167.728$ \\
\hline 10 & $\mathrm{R} \$ 0$ & $R \$ 155.654$ & $-R \$ 5.749$ & $R \$ 149.905$ & $R \$ 537.352$ & $\mathrm{R} \$ 92.029$ & $R \$ 259.757$ \\
\hline 11 & $\mathrm{R} \$ 0$ & $R \$ 166.550$ & $-R \$ 5.749$ & $R \$ 160.801$ & $R \$ 698.152$ & $\mathrm{R} \$ 94.017$ & $\mathrm{R} \$ 353.773$ \\
\hline 12 & $\mathrm{R} \$ 0$ & $R \$ 178.208$ & $-R \$ 5.749$ & $\mathrm{R} \$ 172.459$ & $\mathrm{R} \$ 870.611$ & $R \$ 96.032$ & 449.805 \\
\hline 13 & $\mathrm{R} \$ 0$ & $R \$ 190.683$ & 5.749 & $\mathrm{R} \$ 184.934$ & $\mathrm{R} \$ 1.055 .545$ & $\mathrm{R} \$ 98.074$ & 547.879 \\
\hline 14 & $\mathrm{R} \$ 0$ & $\mathrm{R} \$ 204.031$ & 5.749 & $\mathrm{R} \$ 198.281$ & $\mathrm{R} \$ 1.253 .826$ & $R \$ 100.146$ & $\mathrm{R} \$ 648.025$ \\
\hline 15 & $\mathrm{R} \$ 0$ & $R \$ 218.313$ & $-R \$ 5.749$ & $R \$ 212.564$ & $\mathrm{R} \$ 1.466 .390$ & $\mathrm{R} \$ 102.247$ & $\mathrm{R} \$ 750.272$ \\
\hline 16 & $\mathrm{R} \$ 0$ & $R \$ 233.595$ & $-R \$ 5.749$ & $\mathrm{R} \$ 227.845$ & $\mathrm{R} \$ 1.694 .235$ & $\mathrm{R} \$ 104.379$ & $\mathrm{R} \$ 854.650$ \\
\hline 17 & $\mathrm{R} \$ 0$ & $R \$ 249.946$ & $-R \$ 5.749$ & $\mathrm{R} \$ 244.197$ & $\mathrm{R} \$ 1.938 .432$ & $R \$ 106.542$ & $\mathrm{R} \$ 961.193$ \\
\hline 18 & $\mathrm{R} \$ 0$ & $\mathrm{R} \$ 267.443$ & $-R \$ 5.749$ & $R \$ 261.693$ & $\mathrm{R} \$ 2.200 .126$ & $R \$ 108.739$ & $R \$ 1.069 .932$ \\
\hline 19 & $\mathrm{R} \$ 0$ & $\mathrm{R} \$ 286.164$ & $-R \$ 5.749$ & $\mathrm{R} \$ 280.414$ & $R \$ 2.480 .540$ & $\mathrm{R} \$ 110.969$ & $\mathrm{R} \$ 1.180 .901$ \\
\hline 20 & $\mathrm{R} \$ 0$ & $R \$ 306.195$ & $-\mathrm{R} \$ 5.749$ & $\mathrm{R} \$ 300.446$ & $\mathrm{R} \$ 2.780 .986$ & $\mathrm{R} \$ 113.235$ & $\mathrm{R} \$ 1.294 .136$ \\
\hline 21 & $\mathrm{R} \$ 0$ & $\mathrm{R} \$ 327.629$ & $-R \$ 5.749$ & $\mathrm{R} \$ 321.879$ & $\mathrm{R} \$ 3.102 .865$ & $\mathrm{R} \$ 115.536$ & $\mathrm{R} \$ 1.409 .672$ \\
\hline 22 & $\mathrm{R} \$ 0$ & $R \$ 350.563$ & $-R \$ 5.749$ & $\mathrm{R} \$ 344.813$ & $\mathrm{R} \$ 3.447 .679$ & $\mathrm{R} \$ 117.874$ & $\mathrm{R} \$ 1.527 .547$ \\
\hline 23 & $\mathrm{R} \$ 0$ & $R \$ 375.102$ & $-\mathrm{R} \$ 5.749$ & $R \$ 369.353$ & $\mathrm{R} \$ 3.817 .031$ & $R \$ 120.251$ & $\mathrm{R} \$ 1.647 .797$ \\
\hline 24 & $\mathrm{R} \$ 0$ & $R \$ 401.359$ & $-R \$ 5.749$ & $\mathrm{R} \$ 395.610$ & $\mathrm{R} \$ 4.212 .642$ & $R \$ 122.666$ & $\mathrm{R} \$ 1.770 .463$ \\
\hline 25 & $\mathrm{R} \$ 0$ & $\mathrm{R} \$ 429.454$ & $-R \$ 5.749$ & $\mathrm{R} \$ 423.705$ & $\mathrm{R} \$ 4.636 .347$ & $\mathrm{R} \$ 125.121$ & $\mathrm{R} \$ 1.895 .585$ \\
\hline
\end{tabular}

Fonte: Dados do Autor.

Observa-se ainda que no final do vigésimo quinto ano o valor presente líquido será de $R \$ 1.895 .585,00$. Portanto, além do projeto pagar o investimento inicial e render uma TMA de 5,00\% a.a., irá proporcionar um ganho de mais de $R \$ 1,8$ milhão. A TIR é obtida através da Equação 01 apresentada no Quadro 1, ao igualar

R. gest. sust. ambient., Florianópolis, v. 10, n. 2, p. 84-104, jun. 2021. 
- VPL a zero, e para este projeto é de 20,29\%, portanto, superior à TMA. A economia na geração de energia acumulada anualmente pelo sistema solar, ao final dos 25 anos, é de $R \$ 5.355 .011,00$.

Desta forma, conclui-se que, para a TMA estipulada, o sistema proposto de energia solar fotovoltaica como alternativa de diversificação da matriz energética bem como a redução de custos com energia elétrica é viável para o período analisado, considerando os dados da pesquisa e do projeto. A importância da adoção de tal sistema gerador de energia solar fotovoltaica para a sustentabilidade vai muito além do benefício econômico-financeiro. A dimensão ambiental também será muito beneficiada.

\section{CONSIDERAÇÕES FINAIS}

O presente estudo teve como objetivo analisar a viabilidade econômicofinanceira da instalação de um sistema gerador de energia solar fotovoltaica para reduzir custos e diversificar a matriz energética em uma Instituição de Ensino Superior (IES) no Estado do Ceará. Para tanto, foi realizada uma pesquisa exploratória em uma IES no Estado do Ceará. Para verificar a viabilidade econômico-financeira da energia solar fotovoltaica foram tomadas em consideração as análises de payback descontado, valor presente líquido e taxa interna de retorno.

Os resultados confirmaram que o projeto da instalação de energia solar fotovoltaica, além de ser uma alternativa para reduzir custos e propiciar a ampliação da matriz energética, é viável considerando os dados projetados, proporcionando em um período de 25 anos um VPL de $R \$ 1.895 .585,00$. Este artigo limita-se a analisar apenas a tecnologia aqui proposta, a de geração de energia solar fotovoltaica. Considerando que existem diversas outras tecnologias, há possibilidade de diversificar mais ainda a matriz energética do campus da IES em estudo. Entre as tecnologias, destaca-se a eólica, que é amplamente utilizada no país. Como limitação técnica, está o fato de se considerar neste estudo o cenário de consumo energético atual do campus, em que pese a possibilidade de haver uma expansão na estrutura acadêmica e predial, com a oferta de novos cursos, e possibilidade de funcionamento no horário noturno, por exemplo. Esse fato demandaria um aumento no consumo de energia do campus, que consequentemente demandaria mais geração de energia por parte do sistema fotovoltaico.

R. gest. sust. ambient., Florianópolis, v. 10, n. 2, p. 84-104, jun. 2021. 
Do ponto de vista ambiental, este estudo pode ser ampliado para os demais campi da IES, tendo em vista a contribuição para um ambiente mais sustentável. Do ponto de vista econômico, evidencia-se uma maior eficiência na gestão dos recursos financeiros públicos. Também poderá servir de base para as demais IES localizadas na mesma região deste campus, visto que os dados de geração de energia são similares devido à proximidade geográfica. Assim, para pesquisas futuras, sugere-se a expansão do debate nas esferas social, acadêmica e científico, realizando investigação sobre o uso da energia solar fotovoltaica nas Instituições de Ensino Superior da Região Metropolitana do Cariri.

\section{REFERÊNCIAS}

ACOSTA, Alberto. O bem viver: uma oportunidade para imaginar outros mundos. Tradução de Tadeu Breda. São Paulo: Autonomia Literária / Elefante, 2016.

AGÊNCIA NACIONAL DE ENERGIA ELÉTRICA (Brasil). Micro e minigeração distribuída: sistema de compensação de energia elétrica. 2. ed. Brasília: ANEEL, 2016.

AGÊNCIA NACIONAL DE ENERGIA ELÉTRICA (Brasil). Resolução Normativa N. 235 de Novembro de 2006. Disponível em:

<http://www.aneel.gov.br/cedoc/ren2006235.pdf.> Acesso em: 17 mai. 2021.

ALBUQUERQUE, Mayane Karen de Oliveira. Estudo de viabilidade para implementação de um sistema fotovoltaico em um parque de abastecimento de aeronaves em Fortaleza/CE. 2020. 103 p. Trabalho de Conclusão de Curso (Graduação em Engenharia Elétrica) - Centro de Tecnologia, Universidade Federal do Ceará, Fortaleza, 2020.

ASSAF NETO, Alexandre. Matemática Financeira e suas Aplicações. 9. ed. São Paulo: Editora Atlas, 2007.

BANCO CENTRA DO BRASIL. Taxa SELIC. 2021a. Disponível em: <https://www.bcb.gov.br/controleinflacao/taxaselic.> Acesso em: 8 mai. 2021.

BANCO CENTRA DO BRASIL. Taxas de juros básicas - Histórico. 2021b.

Disponível em: <https://www.bcb.gov.br/controleinflacao/historicotaxasjuros.> Acesso em: 1 mai. 2021.

BARBOSA, Wilson Pereira; AZEVEDO, Abílio César Soares de. Geração distribuída: vantagens e desvantagens. II Simpósio de Estudos e Pesquisas em Ciências Ambientais na Amazônia. Manaus, 2013.

R. gest. sust. ambient., Florianópolis, v. 10, n. 2, p. 84-104, jun. 2021. 
BRASIL. Ministério de Minas e Energia. Empresa de Pesquisa Energética. Balanço Energético Nacional 2019: Ano base 2018. Relatório final / Ministério de Minas e Energia. Empresa de Pesquisa Energética - Rio de Janeiro: EPE, 2019.

BRASIL. Ministério da Educação. Resumo Técnico do Censo da Educação Superior 2017. Brasília: Instituto Nacional de Estudos e pesquisas Educacionais Anísio Teixeira, 2019.

BURGER, Regiane (org.). Ciências do ambiente. Rio de Janeiro: Editora Universidade Estácio de Sá, 2014.

CASTANHEIRA, Nelson Pereira. Cálculo aplicado à gestão e aos negócios [livro eletrônico]; Curitiba: Intersaberes, 2016.

CERTO, Samuel C.; PETER, John Paul. Administração estratégica: planejamento e implantação da estratégia. 2. ed. São Paulo: Pearson Education do Brasil, 2005.

CONTI, Mylena Machado. Estudo da viabilidade de implantação de um sistema fotovoltaico em uma instituição de ensino através da engenharia da sustentabilidade. 2021. 122 p. Monografia (Graduação em Engenharia de Produção) - Instituto de Ciências Exatas e Aplicadas, Universidade Federal de Ouro Preto, João Monlevade, 2021.

CRESESB. Centro de Referência para as Energias Solar e Eólica Sérgio S. de Brito. Potencial Solar - SunData. 2020b. Disponível em:

<http://www.cresesb.cepel.br/index.php?section=sundata>. Acesso em: 13 abr. 2020.

DADALTO, Elder Antonio. Utilização da energia solar para aquecimento de água pela população de baixa renda domiciliar em habitações populares. Vitória: Escola de Engenharia da UFMG, 2008.

ELISARDO, Alax; WIPPEL, Bruno Eduardo. Estudo de projeto de sistemas fotovoltaico voltado a mini e micro geração: para residência conectada a rede. Engenharia Elétrica-Pedra Branca, 2018.

EMPRESA DE PESQUISA ENERGÉTICA (Brasil). Balanço Energético Nacional 2019: Ano base 2018 / Empresa de Pesquisa Energética - Rio de Janeiro: EPE, 2019.

FERNANDES, José Eduardo Stort; SILVA, Arquimedes Lopes da; BELCHIOR, Fernando Nunes. Análise da viabilidade econômica e técnica para implantação de sistemas fotovoltaicos em unidades consumidoras do Tribunal de Justiça do Estado de Goiás. CEEL. Universidade Federal de Uberlândia, Uberlândia, 2020.

FONSECA, Yonara Daltro da; BRUNI, Adriano Leal. Técnicas de avaliação de investimentos: uma breve revisão da literatura.

<http://repositorio.ufba.br/ri/handle/ri/25449> Acesso em: 09 mai. 2020.

GITMAN, Lawrence J. Princípios da administração financeira. 10. ed. Porto Alegre: Bookman, 2004.

R. gest. sust. ambient., Florianópolis, v. 10, n. 2, p. 84-104, jun. 2021. 
GONZÁLEZ, Mario Orestes Aguirre; GONÇALVES, Joeberson S.; VASCONCELOS, Rafael M. (2016) Sustainable development: case study in theimplementation of renewable energy in Brazil. Journal of Cleaner Production 142: 461-475

GUANGUL, Fiseha Mekonnen; CHALA, Girma. Solar energy as renewable energy source: swot analysis. $4^{\text {th }} \mathrm{MEC}$ International conference on big data and smart city. 2019.

HOBMEIR, Liziane; TRINDADE, Eduardo Marques. Ferramenta para estudo da viabilidade econômica de implantação de painéis fotovoltaicos em indústrias. Institutos Lactec. Unicuritiba, 2016.

LANDEIRA, Juan Lourenço Fandino. Análise técnico-econômica sobre a viabilidade de implantação de sistemas de geração fotovoltaica distribuída no Brasil. Rio de Janeiro: UFRJ/COPPE, 2013.

LEMES JUNIOR, Antonio Barbosa; CHEROBIM, Ana Paula; RIGO, Claudio Miessa. Administração financeira: princípios, fundamentos e práticas brasileiras. Rio de Janeiro: Elsevier, 2002.

LIMA, Mirelle Tainá Vieira et al. Índice de desenvolvimento regional sustentável aplicado aos municípios da região metropolitana do cariri. Revista Brasileira de Gestão e Desenvolvimento Regional, v. 17, n. 1, 2021.

MACHADO, Carolina Tomaz; MIRANDA, Fabio da Silva. Energia solar fotovoltaica: uma breve revisão. Revista virtual de química, Vol. 7, No. 1. PP. 126-143. 2015.

MAGNUS, Guilherme Hendler. Energia renovável: sistema fotovoltaico como alternativa para residências em Criciúma-SC. 2019. 27 p. Trabalho de conclusão de curso (Graduação em Ciências Contábeis). Universidade do Extremo Sul Catarinense, Criciúma, 2019.

MELO, Thiago Torres da Paz. Dimensionamento do sistema fotovoltaico on-grid: estudo de caso de uma residência unifamiliar na cidade de Cruz das Almas-BA. 2020. 50 p. Trabalho de conclusão de curso (Graduação em Engenharia Civil). Faculdade Maria Milza, Governador Mangabeira, 2020.

MORIOKA, Sandra Naomi; BOLIS, Ivan; SZNELWAR, Laerte Idal. When sustainable development risks losing its meaning. Delimiting the concept with a comprehensive literature review and a conceptual model. Journal of Cleaner Production, v. 83, p. 7-20, 2014.

NAKABAYASHI, Rennyo Kunizo. Microgeração fotovoltaica no Brasil: condições atuais e perspectivas futuras. 2014. Dissertação (Mestrado em Ciências). Universidade de São Paulo, São Paulo, 2014.

NATIONAL AERONAUTICS AND SPACE ADMINISTRATION. The Balance of Power in the Earth-Sun System. NASA Facts. 2005. Disponível em:

R. gest. sust. ambient., Florianópolis, v. 10, n. 2, p. 84-104, jun. 2021. 
$<$ https://www.nasa.gov/pdf/135642main_balance_trifold21.pdf>. Acesso em: 12 nov. 2020.

OYEDEPO, Sunday Olayinka; et al. Assessment of Energy Saving Potentials in Covenant University, Nigeria. Energy Engineering: Journal of the Association of Energy Engineering, 113(3),74-83. 2016.

PEREIRA, Enio Bueno et al. Atlas brasileiro de energia solar. 2. ed. São José dos Campos: INPE, 2017.

PHILIPPI JR, Arlindo; REIS, Lineu Belico dos. Energia e sustentabilidade. Barueri, Manole: 2016.

SACHS, Ignacy. Caminhos para o desenvolvimento sustentável. Rio de Janeiro: Garamond, 2002.

SAMARA, Beatriz Santos; BARROS, José Carlos. Pesquisa de marketing: conceitos e metodologia. 4. ed. São Paulo: Pearson Prentice Hall, 2007.

SEIFFERT, Mari Elizabete Bernardini. Gestão ambiental: instrumentos, esferas de ação e educação ambiental. Ed. Atlas, 2011.

SILVA, Rutelly Marques. Energia Solar no Brasil: dos incentivos aos desafios. Brasília: Núcleo de Estudos e Pesquisas/CONLEG/Senado, Fevereiro/2015 (Texto para Discussão nํ166).

SOUZA, Gabriela Romana; DA PENHA, Roberto Silva. Viabilidade Econômica de um Projeto de Investimento de Energia Fotovoltaica. RAGC, v. 8, n. 35, 2020.

TIBA, Sofien; BELAID, Fateh. Modeling the nexus between sustainable development and renewable energy: the african perspectives. Journal of Economic Surveys, 35(1), 307-329. doi:10.1111/joes.12401. 2021.

UHL, Christopher; ANDERSON, Amy. Green destiny: universities leading the way to a sustainable future. BioScience, Vol. 51, pp. 36-42. 2001.

WAAS, Tom, VERBRUGGEN Aviel; WRIGHT, Tarah. University research for sustainable development: definition and characteristics explored. Journal of Cleaner Production. 18, 629-636. 2010.

ZHANG, Dong et al. Economic assessment and regional adaptability analysis of CCHP system coupled with biomass-gas based on year-round performance. Sustainable Energy Technologies and Assessments, v. 45, p. 101141. 2021.

R. gest. sust. ambient., Florianópolis, v. 10, n. 2, p. 84-104, jun. 2021. 